\title{
DISTORTION INEQUALITIES FOR RUSCHEWEYH DERIVATIVES
}

\author{
Shigeyoshi Owa AND H. M. SRIVASTAVA
}

Abstract. Let $\mathscr{A}$ denote the class of functions $f(z)$ which are analytic in the open unit disk $\mathscr{U}$ with $f(0)=0$ and $f^{\prime}(0)=1$. For $f(z) \in \mathscr{A}$, the Ruscheweyh derivative of order $\lambda$ is denoted by $\mathscr{D}^{\lambda} f(z)$. The object of the present paper is to derive several distortion inequalities involving $\mathscr{D}^{\lambda} f(z)$ for certain classes of univalent functions $f(z)$ by applying known properties of generalized hypergeometric functions.

Mathematics subject classification (1991): Primary 30C45; Secondary 33C05, 33C20.

Key words and phrases: Analytic functions, univalent functions, Ruscheweyh derivatives, starlike functions, convex functions, generalized hypergeometric functions, Pochhammer symbol, distortion inequalities, Hadamard product (or convolution).

\section{REFERENCES}

[1] M.S. RoberTson, On the theory of univalent functions, Ann. of Math 37 (1936), 374-408.

[2] St. Ruscheweyh, New criteria for univalent functions, Proc. Amer. Math. Soc 49 (1975), 109-115.

[3] H.M. SRIVASTAVA, Generalized hypergeometric functions with integral parameter differences, Nederl. Akad. Wetensch. Indag. Math 35 (1973), 38-40. 